\title{
Controlling the electronic and physical coupling on dielectric thin films
}

\author{
Philipp Hurdax ${ }^{1}$, Michael Hollerer ${ }^{1}$, Larissa Egger ${ }^{1}$, Georg Koller ${ }^{1}$, Xiaosheng Yang ${ }^{2,3,4}$, \\ Anja Haags ${ }^{2,3,4}$, Serguei Soubatch ${ }^{2,3}$, Frank Stefan Tautz ${ }^{2,3,4}$, Mathias Richter ${ }^{5}$, \\ Alexander Gottwald ${ }^{5}$, Peter Puschnig ${ }^{1}$, Martin Sterrer ${ }^{1}$ and Michael G. Ramsey ${ }^{*}$
}

\section{Full Research Paper}

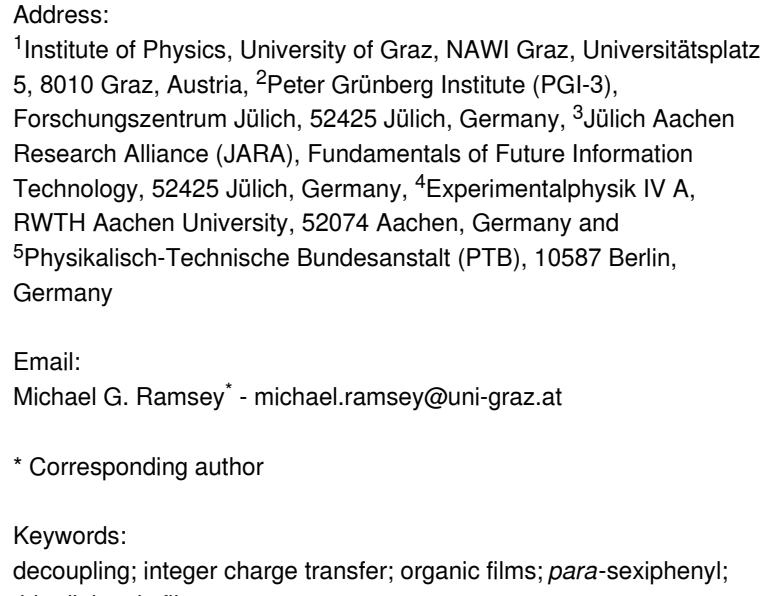

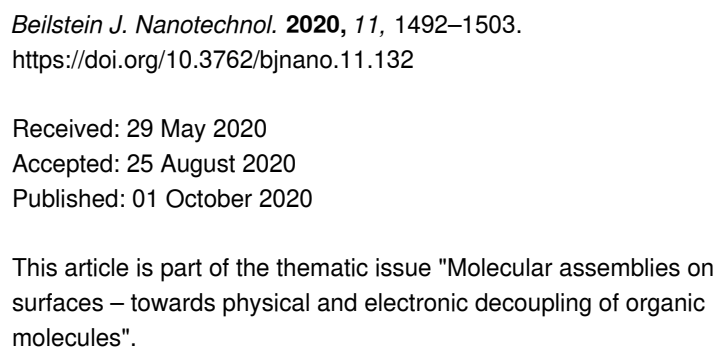

Guest Editor: M. Stöhr

(C) 2020 Hurdax et al.; licensee Beilstein-Institut. License and terms: see end of document.

\begin{abstract}
Ultrathin dielectric/insulating films on metals are often used as decoupling layers to allow for the study of the electronic properties of adsorbed molecules without electronic interference from the underlying metal substrate. However, the presence of such decoupling layers may effectively change the electron donating properties of the substrate, for example, by lowering its work function and thus enhancing the charging of the molecular adsorbate layer through electron tunneling. Here, an experimental study of the charging of para-sexiphenyl (6P) on ultrathin $\mathrm{MgO}(100)$ films supported on $\mathrm{Ag}(100)$ is reported. By deliberately changing the work function of the $\mathrm{MgO}(100) / \mathrm{Ag}(100)$ system, it is shown that the charge transfer (electronic coupling) into the 6P molecules can be controlled, and 6P monolayers with uncharged molecules (Schottky-Mott regime) and charged and uncharged molecules (Fermi level pinning regime) can be obtained. Furthermore, it was found that charge transfer and temperature strongly influence the orientation, conformation, and wetting behavior (physical coupling) of the $6 \mathrm{P}$ layers on the $\mathrm{MgO}(100)$ thin films.
\end{abstract}

\section{Introduction}

Since the first scanning tunneling microscope (STM) imaging of the highest occupied molecular orbital (HOMO) and the lowest unoccupied molecular orbital (LUMO) of pentacene
(5A) on $\mathrm{NaCl} / \mathrm{Cu}(111)$ was performed [1], the concept of decoupling molecules from metal substrates with large bandgap dielectric films has become widely accepted. Although such 
systems have become a rich field of research, particularly in the scanning probe microscopy community, it is often forgotten that the wide bandgap insulating layer is not a sufficient condition for decoupling. Although it reduces wave function overlap with the substrate, it can in fact promote charge transfer via tunneling. The determining factor is the energy level alignment of the frontier orbitals of the adsorbate relative to the Fermi level $\left(E_{\mathrm{F}}\right)$ of the underlying metal $[2,3]$. As dielectric films can significantly reduce the work function, principally due to Pauli repulsion (pushback) at the metal interface, adsorbates of sufficiently high electron affinity (EA) will become negatively charged via tunneling from the underlying metal. This was predicted by Pacchioni et al. [4-6] and either inferred or observed for adsorbates ranging from metal atoms $[7,8]$ and small molecules $[9,10]$ to larger $\pi$-conjugated molecules $[11$ 13].

This phenomenon has been comprehensively analyzed for $5 \mathrm{~A}$ on epitaxial $\mathrm{MgO}(100) / \mathrm{Ag}(100)$, in which orbital-resolved STM and photoemission tomography (PT) have enabled the quantification of both the charge on individual molecules and the number of charged molecules in the 5A monolayer (ML) [14]. For 5A MLs on regularly grown epitaxial $\mathrm{MgO}(100)$ films, all molecules appear to be charged. Orbital-resolved STM reveals the LUMO both above and below the Fermi level, with a large gap between a singly occupied molecular orbital (SOMO) and a singly unoccupied molecular orbital (SUMO). In addition, PT confirms an integer charge transfer, which would be expected to result from tunneling [15]. However, using the ability to tune the $\mathrm{MgO}(100) / \mathrm{Ag}(100)$ work function after $\mathrm{MgO}$ film growth $\left(\Phi_{\mathrm{MgO}}\right)$ by changing the composition at the dielectric-metal interface without changing its surface [16-19], it has been recently shown that two distinct adsorption regimes exist [20]. On films with a high $\Phi_{\mathrm{MgO}}$, all molecules in the ML remain neutral, whereas on a low- $\Phi_{\mathrm{MgO}}$ substrate, charge transfer is observed. In the former (the "vacuum level alignment" regime) the molecules are electronically decoupled, while in the latter, the electronic levels are tied to the Fermi level of the underlying $\operatorname{Ag}(100)$. The equilibration process for this "Fermi level pinning regime" has been realized by the proportion of charged and neutral molecules coexisting in the first monolayer, as schematically illustrated in Figure 1. In this work function regime, ranging from all the molecules being charged to no molecules being charged, the molecular ML is characterized by a constant pinning work function $\left(\Phi_{\text {pin }}\right)$. The critical substrate work function $\left(\Phi_{\text {crit }}\right)$ for the charge transfer is given by the condition $\Phi_{\mathrm{MgO}}=\Phi_{\text {pin. }}$. On the macroscopic level, the simple relationships between the density of the integer charged molecules, the dielectric thickness $\left(d_{\text {diel }}\right)$, and the change in the work function upon adsorption of the molecules $\left(\Delta \Phi=\Phi_{\mathrm{mol}}-\Phi_{\mathrm{MgO}}\right.$, where $\Phi_{\text {mol }}$ is the final work function after adsorption of the mole- cules) is very well described by electrostatics with a simple capacitor model given by

$$
\Delta \Phi=\frac{\sigma\left(d_{\mathrm{diel}}+d_{0}\right)}{\varepsilon_{0} \varepsilon_{\mathrm{r}}}=\frac{\sigma d_{\mathrm{cs}}}{\varepsilon_{0} \varepsilon_{\mathrm{r}}},
$$

where $\sigma$ is the average charge density in the molecular film, $\varepsilon_{\mathrm{r}}$ is the dielectric constant of the thin film, and $d_{\mathrm{cs}}$ is the distance between the charge in the molecule and its image charge in the metal (i.e., the charge separation distance).

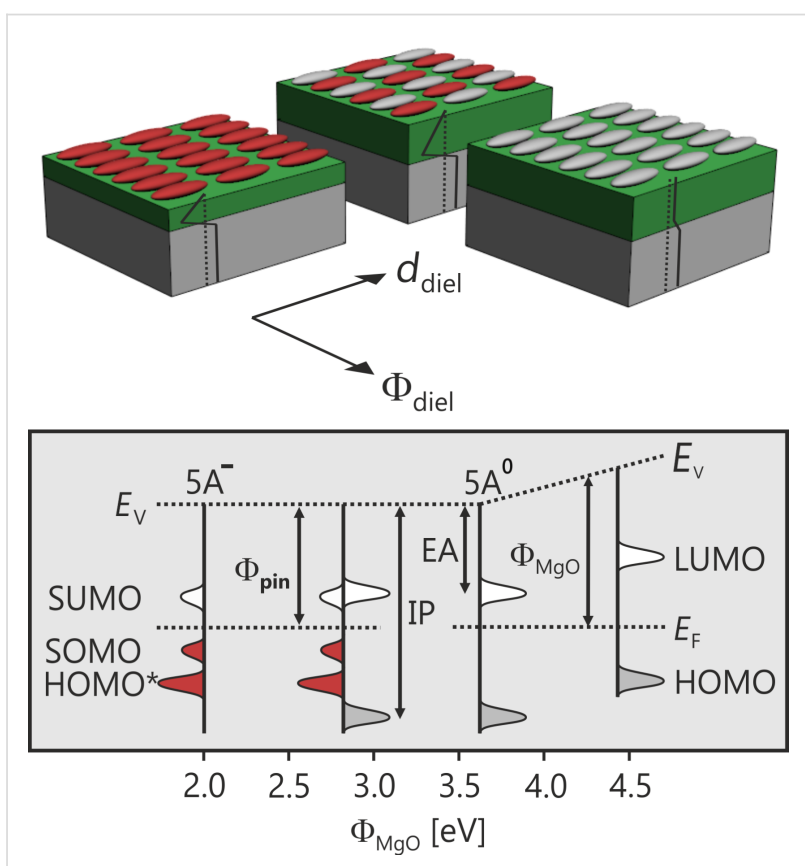

Figure 1: Molecular level schematic of integer charge transfer across dielectric films. Top: The ratio of integer charged (red) and neutral (gray) molecules is determined by the work function of the substrate after dielectric film growth $\left(\Phi_{\text {diel }}\right)$ and the dielectric film thickness $\left(d_{\text {diel }}\right)$, according to the capacitor model. Bottom: Energy level alignment for charged $5 \mathrm{~A}^{-}$and neutral $5 \mathrm{~A}^{0}$ on $\mathrm{MgO}(100) / \mathrm{Ag}(100)$ from the Fermi level pinning to the vacuum level alignment regime. $\mathrm{HOMO}^{*}$ refers to orbitals of charged molecules.

In this report, we demonstrate the robustness of the conclusions drawn from the $5 \mathrm{~A}$ study by considering para-sexiphenyl (6P, $\mathrm{C}_{36} \mathrm{H}_{26}$ ). In contrast to $5 \mathrm{~A}, 6 \mathrm{P}$ is geometrically more flexible, more specifically, it is nonplanar in the gas phase and has a relatively low electron affinity. On the pristine $\operatorname{Ag}(100)$ substrate, 6P simply physisorbs with no evidence of LUMO hybridization, which remains unoccupied [21]. It will be shown that the introduction of the dielectric interlayer can lead to integer charge transfer and electrostatic coupling to this otherwise passive metal surface. Unlike the $5 \mathrm{~A}$ case, $\Phi_{\text {crit }}$ for the charge transfer is not equal to the $\Phi_{\text {pin }}$ for $6 \mathrm{P}$. This is understood in terms of conformational changes in the $6 \mathrm{P}$ and the enhanced physical 
coupling to the substrate induced by the charge transfer. Finally, the charging effect on the thermal dynamics and the stability of the $6 \mathrm{P}$ monolayer are considered.

\section{Results and Discussion \\ Ambivalent behavior of 6P on regularly prepared $\mathrm{MgO}(100) / \mathrm{Ag}(100)$ thin films}

Figure 2 shows STM images of four $6 \mathrm{P} / \mathrm{MgO}(100) / \mathrm{Ag}(100)$ preparations for $\mathrm{MgO}$ films with a thickness of $2 \mathrm{ML}$ (Figure 2a, Figure 2b) and $3 \mathrm{ML}$ (Figure 2c, Figure 2d), and 6P coverage ranging from sub-ML (Figure $2 \mathrm{a}$ ) and close-to-monolayer (Figure 2b, Figure 2c) to $2 \mathrm{ML}$ (Figure 2d). STM shows $6 \mathrm{P}$ molecules arranged in ordered monolayer islands (Figure $2 \mathrm{~b}$, Figure 2c), with their long axes aligned parallel to each other and parallel to the substrate surface. This is typical for $6 \mathrm{P}$ on atomically clean and ordered substrates obtained from bulk oxides to metal substrates [21-25]. In the submonolayer coverage regime (Figure 2a), we find molecules with different orientations coexisting on the surface. Their long axes are aligned along the principal crystallographic directions $[001] /[010]$ and [011]. In the monolayer regime, molecules align either along
[001]/[010] (Figure 2b) or [011] (Figure 2c), while in Figure 2d, where multilayer islands of up to three layers high are present, the molecules in the domains are rotated by $18^{\circ}$ with respect to [011]. Note that on some $\mathrm{MgO}$ preparations, severe island growth was observed, while on others it was less pronounced.

Generally, the molecules appear as rod-like features in STM. A pronounced six-lobe structure with a slight zig-zag appearance is seen in some of the molecules in Figure 2a. This structure is even more evident in the molecules forming the ordered island in Figure 2c. These may be associated with the six phenyl rings of the $6 \mathrm{P}$ molecule presenting a twisted conformation. In the gas phase, $6 \mathrm{P}$ naturally occurs with a torsional angle of $35^{\circ}$ between its phenyl rings [26]. The observation of such a twist on $\mathrm{MgO}(100) / \mathrm{Ag}(100)$ suggests that the interaction between the $6 \mathrm{P}$ molecules and $\mathrm{MgO}$ is very weak. In addition, next to the $6 \mathrm{P}$ molecules with a zig-zag appearance, there are other molecules with 6 lobes, but in a linear arrangement (Figure 2a). A possible explanation for this observation is the charging-induced planarization of 6P. For comparison, on the pristine $\mathrm{Ag}(100)$ substrate, planar 6P molecules are observed in the submonolayer regime despite the lack of any charge transfer, as evi-
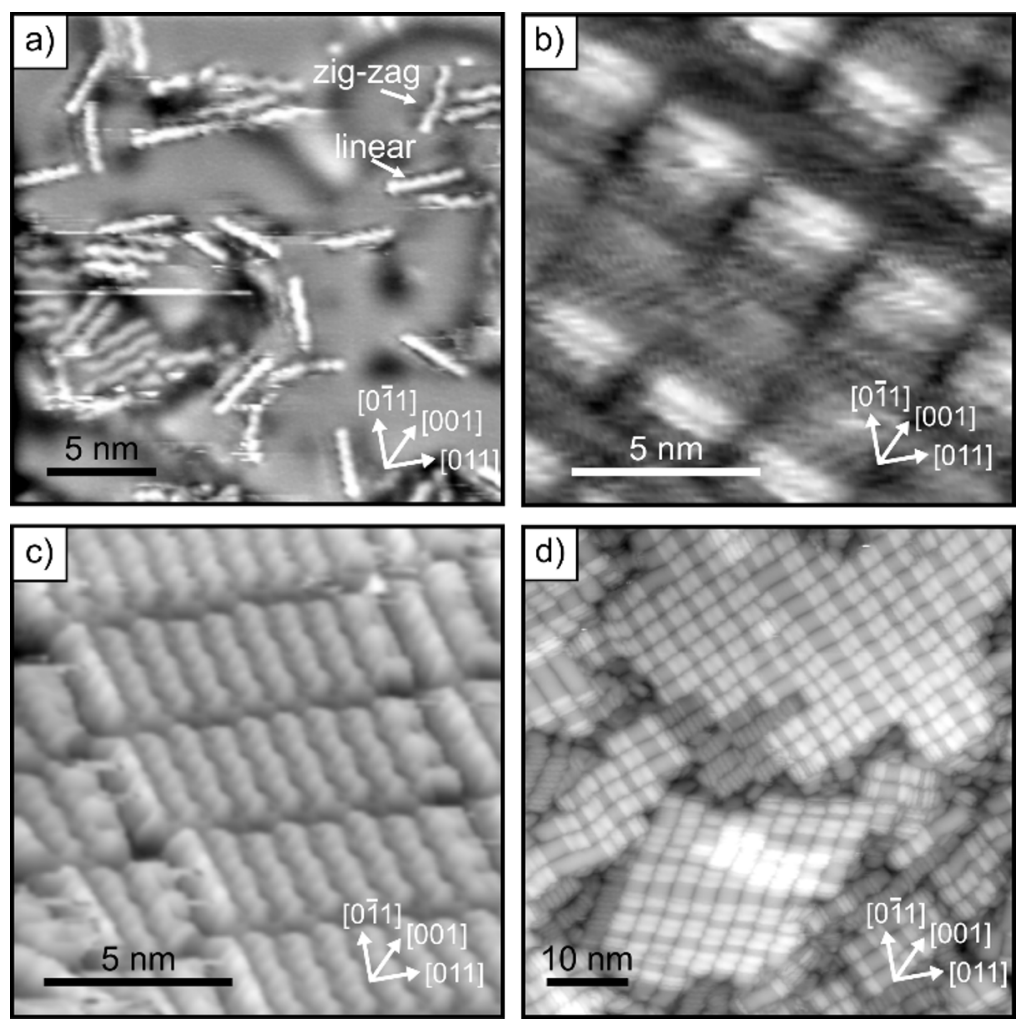

Figure 2: STM images of $6 \mathrm{P}$ deposited on $(\mathrm{a}, \mathrm{b}) 2 \mathrm{ML} \mathrm{MgO}(100) / \mathrm{Ag}(100)$ and $(\mathrm{c}, \mathrm{d}) 3 \mathrm{ML} \mathrm{MgO}(100) / \mathrm{Ag}(100)$. (a) Individual $6 \mathrm{P}$ molecules, $T_{\text {dep, } 6 \mathrm{P}=}$ $-193^{\circ} \mathrm{C}$, size: $25 \mathrm{~nm} \times 25 \mathrm{~nm}, U_{\mathrm{b}}=+0.4 \mathrm{~V}, i_{\mathrm{t}}=25 \mathrm{pA}$; (b,c) $6 \mathrm{P}$ monolayer islands, $T_{\text {dep }, 6 \mathrm{P}}=30^{\circ} \mathrm{C}$, size: $13 \mathrm{~nm} \times 13 \mathrm{~nm}, U_{\mathrm{b}}=+1.4 \mathrm{~V}, i_{\mathrm{t}}=16 \mathrm{pA}(\mathrm{b})$ and $U_{\mathrm{b}}=-0.08 \mathrm{~V}, i_{\mathrm{t}}=140 \mathrm{pA}$ (c). (d) Multilayer $6 \mathrm{P}$ islands, $T_{\text {dep }, 6 \mathrm{P}}=30^{\circ} \mathrm{C}$, size: $60 \mathrm{~nm} \times 60 \mathrm{~nm}, U_{\mathrm{b}}=+1.7 \mathrm{~V}, i_{\mathrm{t}}=30 \mathrm{pA}$. All STM images were recorded at $80 \mathrm{~K}$ 
denced by the absence of an emission from the LUMO in photoemission tomography [21]. This implies that the van der Waals interaction with the metal is sufficient to planarize the molecule. From the STM images shown here of $6 \mathrm{P}$ on the $\mathrm{MgO}(100) / \mathrm{Ag}(100)$ films it is not possible to provide an unambiguous assignment of charged and uncharged molecules, since the orbital resolution has not been achieved in this system. However, the existence of two different 6P appearances (Figure 2a) and monolayer orientations (Figure 2b, Figure 2c) might be related to different charge states of the molecules on the surface.

The images of orbitals of molecules adsorbed on surfaces can also be obtained from the angular intensity distribution in valence band photoemission experiments via PT [27]. When the photoelectron emission angle is converted to momentum, the resulting momentum maps approximately reflect the square of the Fourier transform of the real space orbitals (i.e., the momentum maps image the orbital in the reciprocal space). PT experiments conducted on $6 \mathrm{P}$ monolayers on $\mathrm{MgO}(100) / \mathrm{Ag}(100)$ revealed that films prepared under nominally identical conditions could yield apparently contradicting results. On some preparations, no molecular emissions were observed in the $\mathrm{MgO}$ bandgap, whereas on others, distinctive features appeared in the gap at 0.5 and $2.5 \mathrm{eV}$ below the Fermi level. The momentum maps of these molecular emissions (Figure 3) can be unambiguously assigned to the orbitals and the geometry of the molecules from which they are emitted [28]. The experimental maps in Figure $3 \mathrm{a}$ and Figure $3 \mathrm{~b}$ are in very good agreement with the simulations of the HOMO and LUMO for 6P having two orthogonal orientations. The observation of the LUMO emission clearly shows that charge transfer has occurred.

The azimuthal orientations of the molecules can be derived by comparing the orientations of the molecular emission patterns to the orientation of the emission pattern from the $\operatorname{Ag}(100)$ substrate or from the crystal surface unit cell inferred from low energy electron diffraction (LEED) experiments. This comparison shows that all charged molecules have their long axes aligned $45^{\circ}$ with respect to the close-packed atomic rows of the substrate (i.e., along the $[001] /[010]$ azimuths) in agreement with the orientation of the molecules obtained via STM in Figure 2b.

Significantly, the theoretical maps shown in Figure 3 are for flat lying planar molecules. The photoemission tomography technique is very sensitive to any out-of-plane tilt or twist in 6P [2932]. A nonzero torsional angle leads to orbital periodicity doubling in the real space and orbital periodicity halving in the reciprocal space [23]. It can therefore be concluded that, while neutral molecules can have a twist in different orientations on
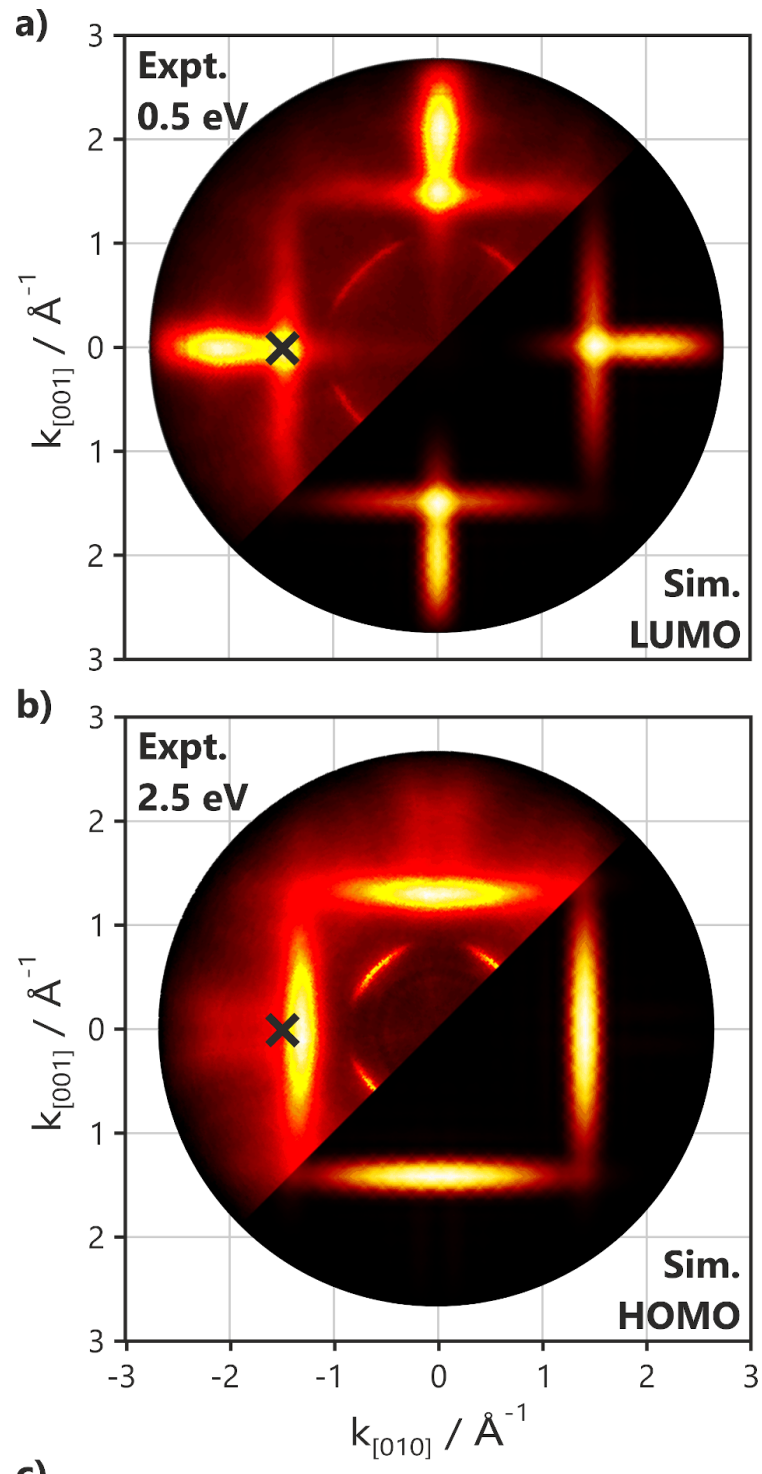

c)

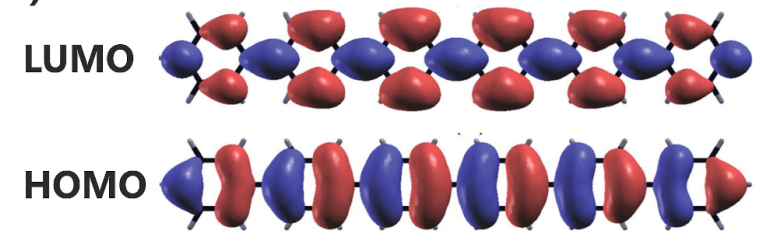

Figure 3: Photoemission momentum maps of $6 \mathrm{P}$ on $\mathrm{MgO}(100) /$ $\mathrm{Ag}(100)$ at energies of (a) $0.5 \mathrm{eV}$ and (b) $2.5 \mathrm{eV}$ below $E_{\mathrm{F}}$, measured with a photon energy of $35 \mathrm{eV}$ and at an incidence angle of $40^{\circ}$. The experimental maps (top left of $a$ and $b$ ) are shown in comparison to the maps calculated from the density functional theory orbitals (c) for two orthogonally oriented planar 6P molecules of the LUMO (bottom right of a) and the HOMO (bottom right of b). The geometry for the maximum LUMO intensity used in the following angle-resolved ultraviolet photoemission spectroscopy experiments is marked with crosses. Deviations from the simulations for isolated molecules are a result of $\mathrm{MgO}$ and $\mathrm{Ag}(100) \mathrm{sp}$-band emissions. Films of $6 \mathrm{P}$ were grown and measured at room temperature. 
the $\mathrm{MgO}$ surface, the molecules that have experienced charge transfer are planarized. Therefore, they lose the $35^{\circ}$ torsional angle between their phenyl rings and align exclusively along $[001] /[010]$. It should be noted that the occupation of the LUMO is predicted to reduce the torsional angle in the molecule, given that it has a bonding character with respect to the phenyl rings (see Figure 3c) [33]. However, this is not a sufficient condition for planarization. Density functional theory (DFT) calculations for the isolated molecule indicate that the torsional angle changes from 35 to only $20^{\circ}$ upon the formation of the anion. Presumably, the electrostatic interaction of the charged molecules with the substrate completes the planarization.

In summary these results suggest that $\mathrm{MgO}$ films prepared under nominally identical conditions can result in either charged or uncharged molecules. While uncharged molecules retain the torsional angle between their phenyl rings, the charge transfer is accompanied by a change in the orientation of the molecules and they become planar. In the following subsection, the critical role of the work function for charging and its influence on the surface wetting capability of the molecules will be highlighted.

\section{Work function control of electronic coupling}

Figure 4 displays angle-resolved ultraviolet photoemission spectroscopy (ARUPS) scans after the 6P molecules were submitted to the same saturation exposure on the same $2 \mathrm{ML}$ $\mathrm{MgO}(100)$ film. The initial work function, $\Phi_{\mathrm{MgO}}$, was tuned to different values. Figure 4a shows wide scans in normal emission, where the $\mathrm{MgO}$ valence band dominates at an energy between 9 and $4 \mathrm{eV}$ below $E_{\mathrm{F}}$.

For $\Phi_{\mathrm{MgO}}$ greater than $2.8 \mathrm{eV}$, there is only a minor decrease in the work function $(<0.1 \mathrm{eV})$ and only a small attenuation in the $\mathrm{MgO}$ emissions of approximately $15 \%$ compared to the clean substrate, suggesting that only a small fraction of the surface is covered with molecules and 3D islands are formed. For $\Phi_{\mathrm{MgO}}$ below $2.8 \mathrm{eV}$, the results change completely. They show the onset of significant substrate attenuation, changes in the spectral lineshape, and an increase in the work function upon 6P deposition. The attenuation behavior suggests that the molecules fully wet the surface only for $\Phi_{\mathrm{MgO}}$ below $2.65 \mathrm{eV}$, where a $60 \%$ reduction in the $\mathrm{MgO}$ intensity is observed. The increase of the work function indicates a charge transfer to 6P. The charge transfer is observed directly by the presence of a LUMO emission seen at high emission angles in Figure 4a. Magnified scans of the $\mathrm{MgO}$ bandgap region in the geometry of maximum LUMO emission intensity, indicated by the crosses in Figure 3, are displayed in Figure $4 \mathrm{~b}$. For $\Phi_{\mathrm{MgO}}$ greater than $2.8 \mathrm{eV}$, no significant orbital emissions are found in the gap, implying that no charged molecules are present on the surface. For $\Phi_{\mathrm{MgO}}$ below $2.8 \mathrm{eV}$, molecular emissions arise at 2.52 and $0.55 \mathrm{eV}$ with respect to $E_{\mathrm{F}}$. Their photoemission energies and angular
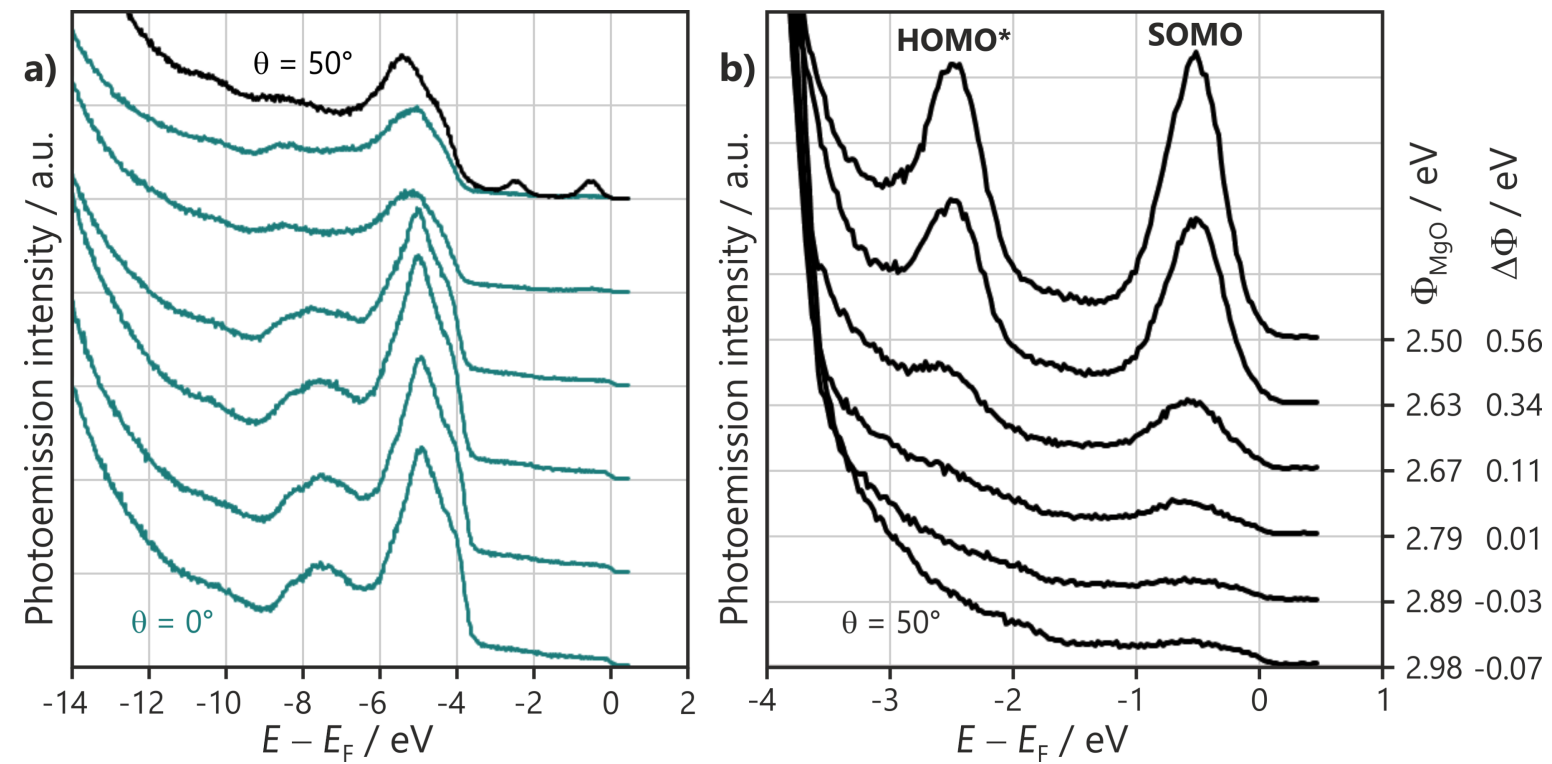

Figure 4: He I ARUPS spectra for different preparations with the same saturation exposure of $6 \mathrm{P}$ (4 $\AA$ ) on the same $2 \mathrm{ML} M g \mathrm{O}$ film tuned to different work functions. $\Phi_{\mathrm{MgO}}$ values and the change in $\Phi$ induced on $6 \mathrm{P}$ exposure $(\Delta \Phi)$ are given on the right. (a) Wide scans at normal emission $\left(\theta=0^{\circ}\right)$ emphasizing the MgO emission region. The black curve shows the ARUPS spectrum measured at $\theta=50^{\circ}$ in the [001] azimuth, where the $6 \mathrm{P}$ HOMO* and $\mathrm{SOMO}$ emissions in the $\mathrm{MgO}$ bandgap region are prominent. (b) Magnification of the $\mathrm{HOMO}^{*}$ and SOMO emissions in the MgO bandgap at $\theta=50^{\circ}$. 
distributions agree with the photoemission tomography of the HOMO and LUMO of the planar 6P seen in Figure 3. Note that the emissions in the $\mathrm{MgO}$ bandgap are due to the orbitals of charged molecules and will be referred to as HOMO* and SOMO henceforth. Neutral molecules are difficult to observe directly via ARUPS, as their HOMO emission lies below the gap in the region of intense $\mathrm{MgO}$ emissions (Figure 5a). The $\mathrm{HOMO}^{*}$ and SOMO intensities increase with decreasing $\Phi_{\mathrm{MgO}}$, indicating an increase in the number of charged molecules. Thus, $\Phi_{\mathrm{MgO}}=2.8 \mathrm{eV}$ is the critical work function value, below which charge transfer into $6 \mathrm{P}$ molecules occurs. The extreme sensitivity to $\Phi_{\mathrm{MgO}}$ shown in Figure 4 explains the apparently ambivalent results obtained from STM and photoemission tomography in the previous section, as the accepted procedure for growing epitaxial $\mathrm{MgO}(100)$ films on $\mathrm{Ag}(100)$ [19,34] produces a scatter of the $\Phi_{\mathrm{MgO}}$ values on either side of the critical work function $\left(\Phi_{\text {crit }}=2.8 \mathrm{eV}\right)$.

Figure 5 shows the evolution of the ARUPS spectra with an increasing 6P dose on an $\mathrm{MgO}(100)$ film with $\Phi_{\mathrm{MgO}}=2.58 \mathrm{eV}$ (i.e., below $\Phi_{\text {crit }}$ ). In the $\mathrm{MgO}$ valence band region (Figure 5a), gradual changes can be observed. In the $\mathrm{MgO}$ bandgap region (magnified in Figure 5b), the intensities of the HOMO* and SOMO increase and reach their maximum value at a $6 \mathrm{P}$ dose between 1 and $1.5 \AA$. The increase in the molecular emission features is accompanied by an increase in the work function $(\Delta \Phi$, values listed on the right). The dose corresponding to a full 6P monolayer is estimated to be approximately $2.8 \AA$ from the attenuation behavior of the valence band of the underlying $\mathrm{MgO}$. The fact that the molecular emissions and $\Phi$ saturate well before the completion of the ML suggests that a significant number of neutral molecules are also present in the ML on this specific $\mathrm{MgO}$ film. A close inspection of Figure 5a shows that the HOMO of the neutral molecules can be seen at $\approx 3.6 \mathrm{eV}$.

In previous research, the charge transfer to $5 \mathrm{~A}$ on $\mathrm{MgO}(100) /$ $\mathrm{Ag}(100)$ was quantified by comparing ARUPS data to predictions made by PT, yielding a LUMO occupation of 1 electron [14]. Following the same approach for $6 \mathrm{P}$, the ratio between the $\mathrm{HOMO}^{*}$ and the SOMO maximum intensity values is predicted to be 0.57 for a singly occupied LUMO. The various 6P preparations yielded intensity ratio values ranging from $0.60-0.75$, suggesting a LUMO occupation of 1.0 to 1.3 electrons. This is consistent with single integer charge transfer, expected for tunneling, when uncertainties in the theoretical description of the photoemission intensities of the simple planewave final-state approximation and in the experimental determination of the peak areas are considered.

\section{The capacitor model and the interlayer dielectric constant}

The behavior observed in the ARUPS series of Figure 4 and Figure 5 is consistent with the predictions from the capacitor model expressed in Equation 1. Figure 6a depicts the relationship between the SOMO intensity $\left(I_{\text {SOMO }}\right)$ and the work function change induced by molecule adsorption $(\Delta \Phi)$, obtained a)

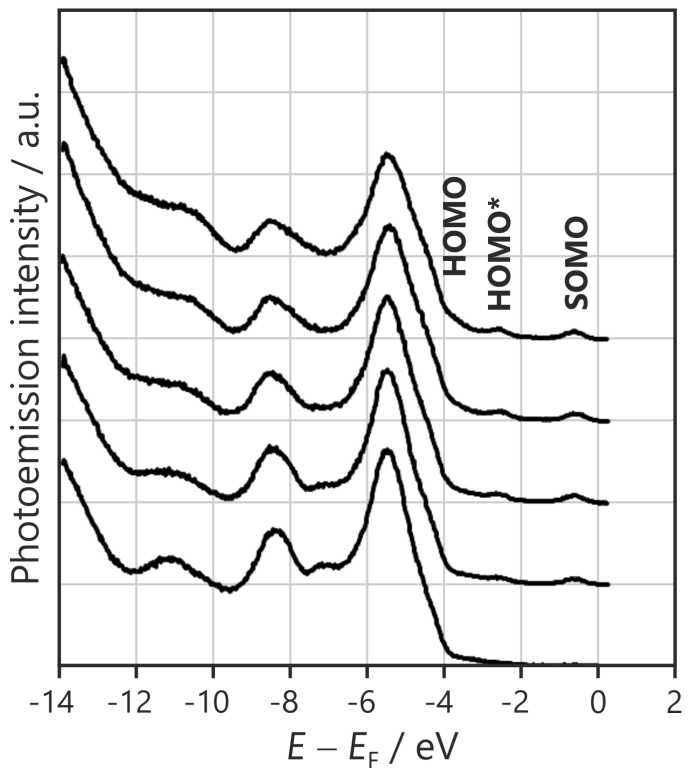

b)

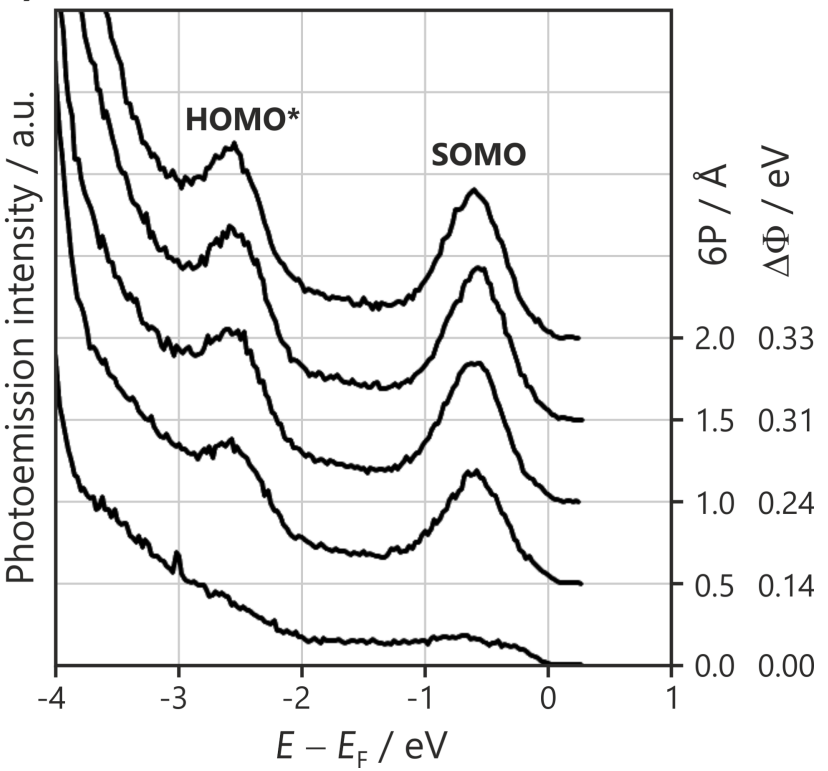

Figure 5: He I ARUPS spectra for a $6 \mathrm{P}$ dosing series on $2 \mathrm{ML} \mathrm{MgO}(100) / \mathrm{Ag}(100)\left(\Phi_{\mathrm{MgO}}=2.58 \mathrm{eV}\right)$ recorded at a takeoff angle of $50^{\circ}$ in the $[001]$ azimuth. (a) Full scan and (b) magnification of the molecular emissions in the MgO bandgap. $6 \mathrm{P}$ exposure and change in the work function upon deposition are indicated on the right. 
from the series of $6 \mathrm{P}$ saturation doses on $\mathrm{MgO}$ films with different $\Phi_{\mathrm{MgO}}$ values. The linear proportionality between $I_{\mathrm{SOMO}}$ and $\Delta \Phi$ agrees with the capacitor model [20], considering that $I_{\text {SOMO }}$ reflects the number of integer charged molecules in the monolayer and thus the charge density $(\sigma)$ on the surface. a)

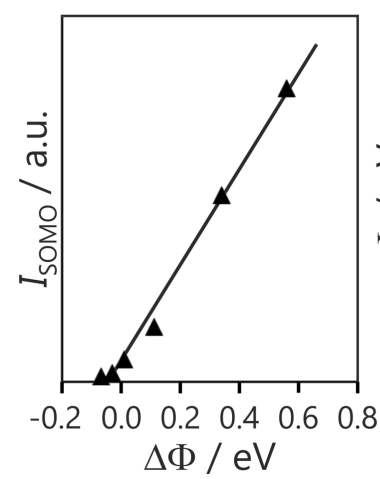

b)

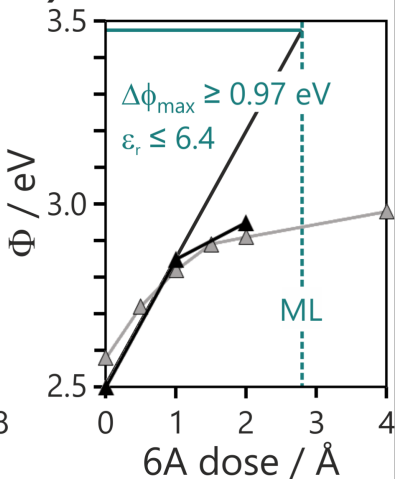

Figure 6: (a) Relation between integrated SOMO intensity and work function change induced by molecule adsorption on $\mathrm{MgO}(100) / \mathrm{Ag}(100)$ $(\Delta \Phi)$ after tuning $\Phi_{\mathrm{MgO}}$ to slightly different values. (b) Work function versus $6 \mathrm{P}$ dose for two different initial work functions. The dose corresponding to a full $\mathrm{ML}$ is indicated by the dashed line.

For the capacitor model to be predictive and quantitative for ultrathin decoupling layers, a realistic value for their dielectric constant $\left(\varepsilon_{\mathrm{r}}\right)$ is required. This can be estimated from a molecular dosing series, such as in Figure 5. Figure 6b shows the increase of $\Phi$ with an increasing 6P dose for two different $\mathrm{MgO}$ films with $\Phi_{\mathrm{MgO}}<\Phi_{\text {crit }}$ values. Initially, $\Phi$ increases rapidly with $6 \mathrm{P}$ exposure since all molecules become charged. The curve then flattens out as $\Phi$ approaches $\Phi_{\text {pin }}$ and additional molecules remain neutral. This is reached at an exposure of $\approx 1.4 \AA$, which is half of the amount required to complete the ML. It can be concluded that on these films there is a 50/50 mix of charged and neutral molecules in the completed monolayer.

Assuming all molecules are charged at low exposure, the $\varepsilon_{\mathrm{r}}$ value can be estimated from the initial slope $(\Delta \Phi / \sigma)$ in Figure 6b, according to:

$$
\varepsilon_{\mathrm{r}}=\frac{\sigma}{\Delta \Phi} \frac{d_{\mathrm{cs}}}{\varepsilon_{0}}
$$

In order to determine the charge density, the knowledge of the density of charged molecules in the monolayer is required. In the case of pentacene, this could be reliably measured with STM, as 5A monolayers with all molecules charged could be produced. The LEED image for 6P films on $\mathrm{MgO}(100) / \mathrm{Ag}(100)$ with a high proportion of charged molecules showed faint superstructure spots, which suggest a later molecular spacing of
6.6 ̊. The van der Waals dimensions of $6 \mathrm{P}(6.7 \times 27.2 \AA)$ [26] are close to the unit cell $(6.35 \times 27.47 \AA)$ which is commensurate with $\mathrm{MgO}(100)$. The area of this unit cell corresponds to a molecular footprint of $173 \AA^{2}$ and gives an upper bound of 6.4 for $\varepsilon_{\mathrm{r}}$. The ordered structure in the STM image of Figure 2b, where molecules do not show a zig-zag structure and are oriented along [11] (like the charged molecules in PT), is consistent with this lateral spacing. However, this structure suggests a slightly longer length of $31 \AA$ for the long unit cell vector. This value yields a molecular area of $197 \AA^{2}$ and an $\varepsilon_{\mathrm{r}}$ of 5.7. This estimate is in agreement with the values for $\varepsilon_{\mathrm{r}}$ (between 5 and 6) obtained for $5 \mathrm{~A} / \mathrm{MgO}(100) / \mathrm{Ag}(100)$ using two different approaches and $\mathrm{MgO}$ thickness values ranging from 2 to $8 \mathrm{ML}$ [20]. This suggests that the effective $\varepsilon_{\mathrm{r}}$ for $\mathrm{MgO}$ thin films is significantly smaller than the bulk value of 9.9 [35].

\section{Critical and pinning work functions}

The rather abrupt changes over a narrow work function range observed for both the charge transfer and wetting behavior for $6 \mathrm{P}$ are in contrast with the $5 \mathrm{~A}$ system. In the latter, the number of charged molecules increases gradually with $\Phi_{\mathrm{MgO}}$ below the critical work function, and a wetting ML is always present, whether or not the charge transfer occurs. Due to the critical role of the work function for charge transfer, it is convenient to plot the work function after saturating the surface with molecules $\left(\Phi_{\mathrm{mol}}\right)$ as a function of $\Phi_{\mathrm{MgO}}$. This is shown in Figure 7 for both $5 \mathrm{~A}$ and $6 \mathrm{P}$ on $2 \mathrm{ML} \mathrm{MgO}(100) / \mathrm{Ag}(100)$. For the $5 \mathrm{~A}$ case, the regimes of vacuum level alignment and Fermi level pinning are clearly expressed: In the vacuum level alignment regime, there is no charge transfer and the final work function closely follows the Schottky-Mott rule in which $\Phi_{\mathrm{mol}}=\Phi_{\mathrm{MgO}}$ (the $45^{\circ}$ line in Figure 7), albeit with a slight reduction in $\Phi$ due to Pauli repulsion (pushback). The Fermi level pinning is characterized by a constant final work function, $\Phi_{\text {pin }}$, and an increase in the number of charged molecules with decreasing $\Phi_{\mathrm{MgO}}$. The number of charged molecules is determined by the potential needed to raise $\Phi$ to $\Phi_{\text {pin }}$ values, whereupon no further charge transfer can occur. It is quite intuitive that $\Phi_{\text {pin }}$ equals the critical work function for the onset of charge transfer, $\Phi_{\text {crit }}$.

However, surprisingly, this is not the case for $6 \mathrm{P}$ as shown in red in Figure 7 . For high $\Phi_{\mathrm{MgO}}$ values, again $\Phi_{\mathrm{mol}}$ is essentially equal to $\Phi_{\mathrm{MgO}}$. The critical work function for the charge transfer (i.e., the point where $\Phi_{\text {mol }}$ increases above the $45^{\circ}$ line) is approximately $2.8 \mathrm{eV}$. This is significantly lower than the value for $5 \mathrm{~A}$, which is expected due to the lower EA of $6 \mathrm{P}$ (theoretical values of $2.39 \mathrm{eV}$ and $1.45 \mathrm{eV}$, respectively, using the B3LYP functional). However, unlike for 5A, as $\Phi_{\mathrm{MgO}}$ decreases further, $\Phi_{\mathrm{mol}}$ does not remain constant but increases above $\Phi_{\text {crit }}$. This reflects the rapid increase in the number of charged molecules observed in Figure 4. Only for 


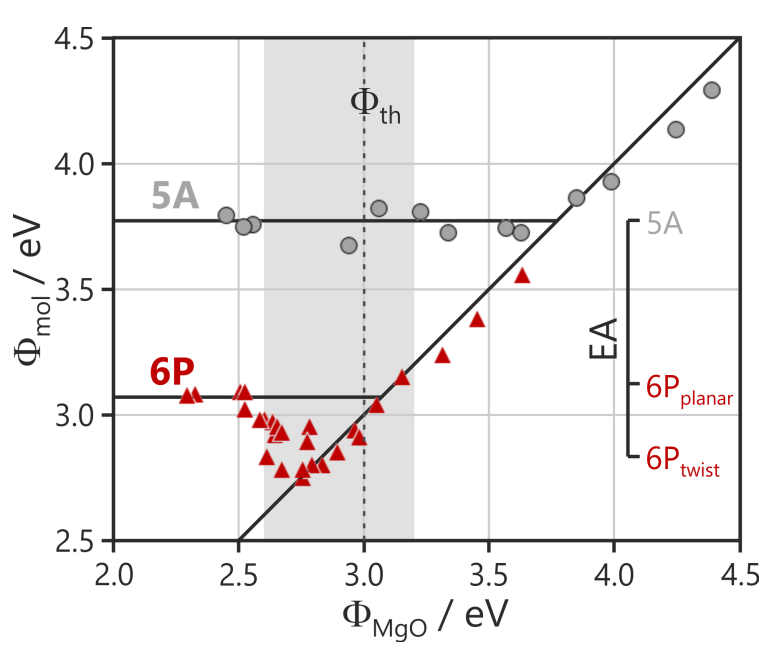

Figure 7: The work function after the deposition of a saturating dose of $5 \mathrm{~A}$ (grey) and $6 \mathrm{P}$ (red) $\left(\Phi_{\mathrm{mol}}\right)$ as a function of the initial work function of a clean $\mathrm{MgO}(100) / \mathrm{Ag}(100)$ substrate $\left(\Phi_{\mathrm{MgO}}\right)$. The line with a slope of unity expresses the Schottky-Mott limit of the vacuum level alignment $\left(\Phi_{\mathrm{mol}}=\Phi_{\mathrm{MgO}}\right)$. The horizontal lines correspond to the pinning work functions of $5 \mathrm{~A}$ and $6 \mathrm{P}$ on $2 \mathrm{ML} \mathrm{MgO}$ films on $\mathrm{Ag}(100)$. The dashed line indicates the theoretical work function for a stoichiometric $\mathrm{MgO}-\mathrm{Ag}(100)$ interface [36], and the shaded gray area indicates the most typical range of work functions obtained with $\mathrm{MgO}$ film growth under nominally identical conditions. The inserted scale shows the theoretical gas-phase electron affinities for $5 \mathrm{~A}$, twisted $6 \mathrm{P}$, and planar $6 \mathrm{P}$ and it has been shifted to align the EA of $5 \mathrm{~A}$ with the measured pinning work function for $5 \mathrm{~A}$.

$\Phi_{\mathrm{MgO}} \leq 2.5 \mathrm{eV}$ the final work function adopts a constant value of $3.07 \mathrm{eV}$, which is the pinning work function of $6 \mathrm{P}$ on $2 \mathrm{ML}$ $\mathrm{MgO}(100) / \mathrm{Ag}(100)$. Thus, for $6 \mathrm{P}, \Phi_{\text {pin }}$ is $0.3 \mathrm{eV}$ higher than $\Phi_{\text {crit }}$

We suggest that this difference is related to the conformational change of $6 \mathrm{P}$ upon charging. $\Phi_{\text {crit }}$ derives from the electron affinity of the neutral, twisted molecule, while $\Phi_{\text {pin }}$ derives from the EA of the planar molecule. The planarization of the molecule will lead to greater orbital overlap between the phenyl rings. This will cause an increase in the energy spread of the $\pi$ bands, resulting in a decrease in the ionization potential and an increase in the electron affinity. Gas phase DFT calculations show that the EA of planar $6 \mathrm{P}(1.74 \mathrm{eV})$ is indeed $0.3 \mathrm{eV}$ higher than the EA of twisted $6 \mathrm{P}(1.45 \mathrm{eV})$. This is in close agreement with the measured difference between $\Phi_{\text {pin }}$ and $\Phi_{\text {crit }}$ seen in Figure 7 , while only a negligible difference in molecular surface dipole is expected between twisted and planar molecules [37]. It should be noted that this alone cannot explain that, depending on the initial work function, either $\Phi_{\text {crit }}$ or $\Phi_{\text {pin }}$ is the decisive work function for charge transfer.

In order to better understand the transition between the two regimes, an alternative representation of the data from Figure 7 is given in Figure 8a. In the latter, the work function change upon 6P deposition as a function of the substrate work function, for various $6 \mathrm{P}$ films on a number of different $2 \mathrm{ML} \mathrm{MgO}$ films is plotted. This is displayed in comparison to the $6 \mathrm{P}$ surface coverage, which is expressed by a wetting parameter in Figure $8 \mathrm{~b}$. The wetting parameter reflects the combined effects of the intensity of all molecular emissions and the suppression of substrate emissions (see Experimental section). The small wetting parameter in the vacuum level alignment regime confirms that molecules do not wet the surface, indicating a low molecule-substrate interaction and 3D island formation.
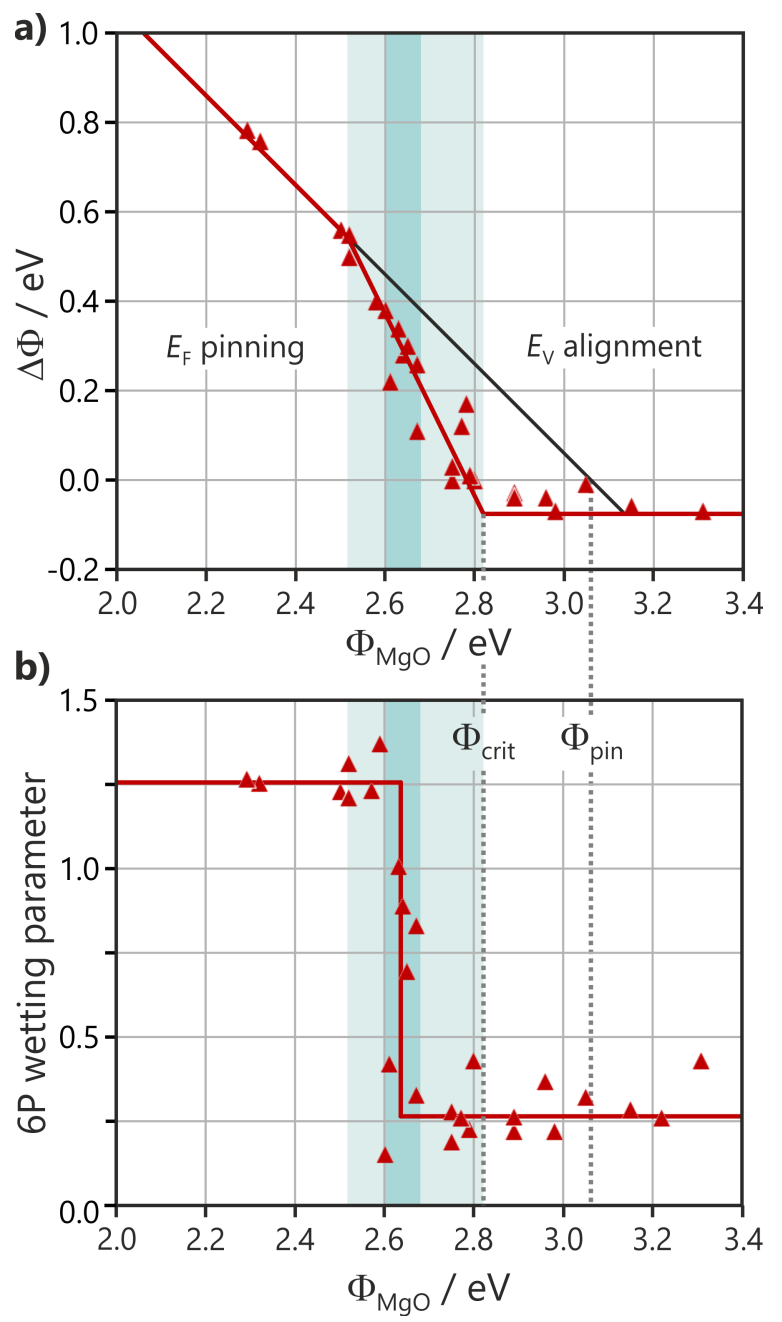

Figure 8: Changes in the charge transfer and wetting behavior of $6 \mathrm{P}$ on $\mathrm{MgO}(100) / \mathrm{Ag}(100)$ as a function of $\Phi_{\mathrm{MgO}}$. (a) Work function change induced by $6 \mathrm{P}$ as an indicator of charge transfer. The black $45^{\circ}$ line corresponds to a constant final work function and expresses the behavior when $\Phi_{\text {pin }}=\Phi_{\text {crit }}$. (b) Wetting parameter as an indicator of the fraction of the substrate covered by $6 \mathrm{P}$.

For $\Phi_{\mathrm{MgO}}$ values between $\Phi_{\text {crit }}$ and $2.52 \mathrm{eV}$, Figure 8a exhibits a steep rise in $\Delta \Phi$ and, therefore, in the number of charged molecules. However, the wetting indicated in Figure $8 \mathrm{~b}$ remains low, until $\Phi_{\mathrm{MgO}}$ reaches approximately $2.64 \mathrm{eV}$, where an 
abrupt discontinuous transition to high wetting occurs. We propose that the critical factor for this phase transition is the density of charged molecules present on the surface. The planarization and electrostatic interaction of the charged molecules with the substrate can be expected to reduce the mobility of the charged molecules. These charged molecules will in turn restrict the free motion of the neutral molecules and, at a critical density, immobilize and reorient them. This wetting transition occurs when $30 \%$ of the molecules are charged, as estimated from $\Delta \Phi$ in the capacitor model, while the final $\Phi_{\text {pin }}$ is established after $60 \%$ of the molecules are charged.

\section{The effect of charge transfer and physical coupling on thermal stability}

We now turn to the role of charge transfer on the physical coupling by considering the thermal stability of the molecular film. This is considered in Figure 9a and Figure $9 \mathrm{~b}$ for a 6P deposit of $4 \AA$ on two $2 \mathrm{ML}$ thick $\mathrm{MgO}$ films for $\Phi_{\mathrm{MgO}}$ values above (Figure 9a) and below (Figure 9b) the critical work function for charge transfer. The graphs show the intensity of the substrate photoemission signal ( $\mathrm{MgO}$ valence band peak intensity in normal emission) and the response of the work function while increasing the temperature. For the high $\Phi_{\mathrm{MgO}}$ film case, where the ARUPS results show no charged molecules on the surface, the temperature increase leads first to an apparently counterintuitive decrease in substrate intensity until the temperature reaches approximately $160{ }^{\circ} \mathrm{C}$. This is followed by an abrupt increase in intensity until the temperature reaches $200{ }^{\circ} \mathrm{C}$. At that point, since the intensity has almost returned to its value prior to the 6P deposition, all the molecules can be considered to be desorbed from the surface. This behavior is mirrored in the work function, which first decreases and then, at $160{ }^{\circ} \mathrm{C}$, increases, returning to its original value by $200{ }^{\circ} \mathrm{C}$. The initial substrate intensity suggests that, at room temperature, only approximately $20 \%$ of the surface is covered by molecules, implying that $3 \mathrm{D}$ islanding occurs. The temperature increase liberates molecules from the islands and allows them to wet the $\mathrm{MgO}$ surface. Right before their desorption at $160{ }^{\circ} \mathrm{C}$, the attenuation of the substrate emission signal suggests a complete surface coverage. The temperature-induced wetting of the surface with neutral molecules is reflected in the pushback-induced lowering of $\Phi$ by $\approx-0.1 \mathrm{eV}$.

The desorption behavior on the low $\Phi_{\mathrm{MgO}}$ film, with up to $80 \%$ of the molecules charged according to the capacitor model (Figure 9b), is quite different. The initial substrate attenuation confirms that the molecules wet the surface at room temperature. There is no significant change in this attenuation until the temperature reaches $160^{\circ} \mathrm{C}$. At that point, the substrate intensity increases as the molecules begin to desorb from the surface. From Figure 9a, we already know that this is the desorption

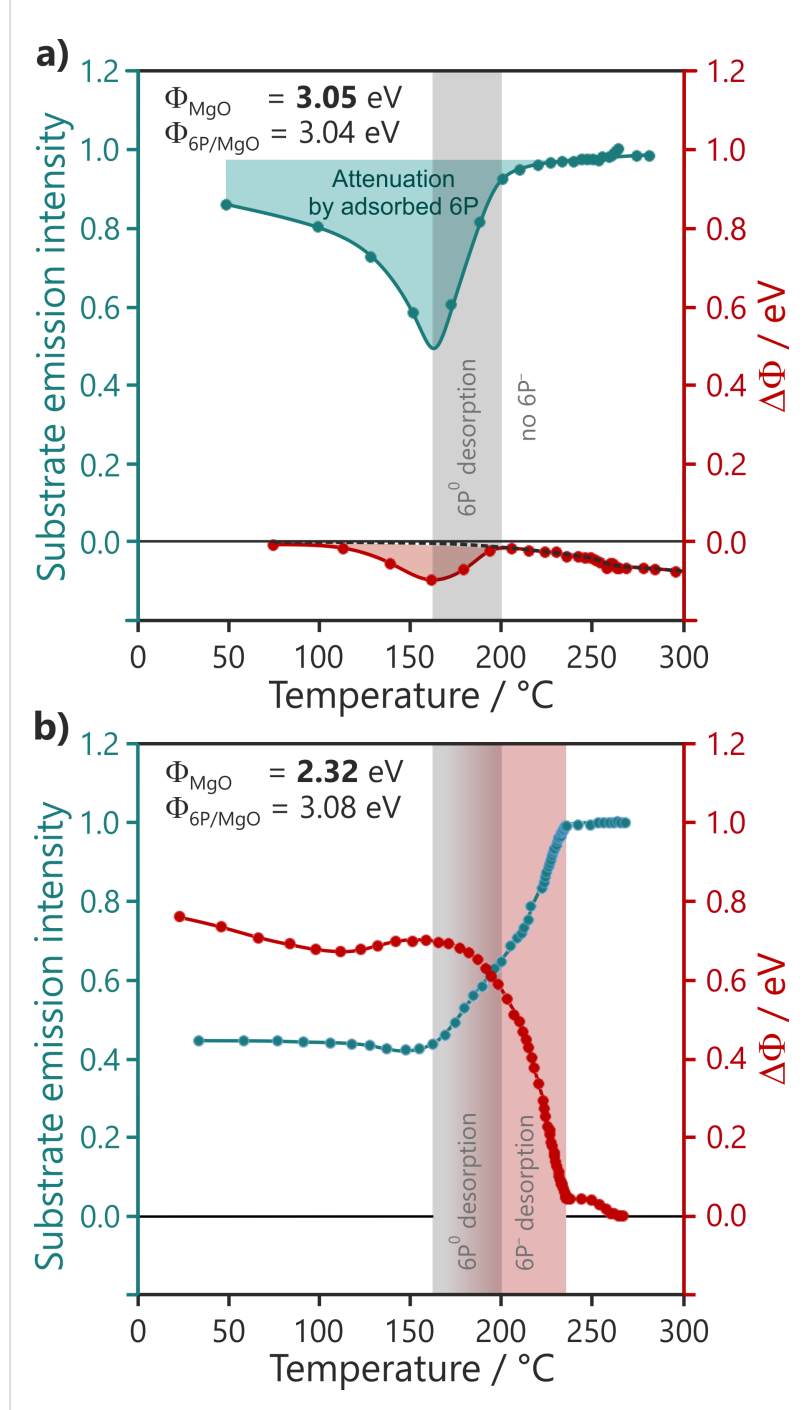

Figure 9: Thermal stability of $6 \mathrm{P}$ films (4 $\AA$ ) on $2 \mathrm{ML}$ of $\mathrm{MgO}$ with work functions (a) above and (b) below the critical work function. The blue curves depict the normalized photoemission intensity of the $\mathrm{MgO}$ valence band relative to the intensity after $6 \mathrm{P}$ film desorption. The red curves depict the change in work function relative to the pristine $\mathrm{MgO}$ film work functions, $\Phi_{\mathrm{MgO}}$.

temperature of neutral molecules. However, on this $\mathrm{MgO}$ film, desorption continues well beyond $200{ }^{\circ} \mathrm{C}$, and the substrate intensity returns to the value corresponding to the clean substrate at $230{ }^{\circ} \mathrm{C}$. It stands to reason that this further increase in the substrate intensity between 200 and $230{ }^{\circ} \mathrm{C}$ is caused by the desorption of the initially charged molecules. This is confirmed by the behavior of the work function in Figure 9b, which shows a concomitant strong decrease in this temperature range. This higher desorption temperature of the charged molecules relative to the neutral molecules can be expected due the electrostatic interaction of the charged molecule with its image charge. It may therefore seem surprising that the onset of the work function decrease and, therefore, the desorption of charged mol- 
ecules happens already within the desorption regime of the neutral molecules. This might be explained by the spontaneous discharging as a result of the thermal energy distribution, enabling desorption as a neutral molecule.

\section{Conclusion}

While charged 6P molecules could not be unambiguously identified by STM, valence band photoemission measurements allowed for their clear observation. Moreover, PT and ARUPS allowed the orientation and number of charged molecules to be quantified. The number of charged molecules and the determination if a given molecule was charged or not were found to be extremely sensitive to the precise work function of the particular $\mathrm{MgO}(100) / \mathrm{Ag}(100)$ film preparation. This was investigated systematically by tuning the work function of a $\mathrm{MgO}(100) /$ $\mathrm{Ag}(100)$ film to values around the critical work function for integer charge transfer to 6P molecules. The simple relationship observed between the number of charged molecules and the work function change on the molecular adsorption proves that the charge transfer is governed by simple electrostatics. This confirms the conclusions drawn from a recent study with pentacene [20] and suggests that the $\varepsilon_{\mathrm{r}}$ of thin dielectrics may be significantly different from the values of their bulk counterparts (e.g., the $\varepsilon_{\mathrm{r}}$ of the $\mathrm{MgO}$ film presented here is approximately half the value of bulk $\mathrm{MgO}$ ).

In contrast to pentacene, $6 \mathrm{P}$ is a geometrically flexible molecule. The charge transfer to $6 \mathrm{P}$ leads to both conformational and orientational changes and has a profound influence on the wetting and thermal stability of the $6 \mathrm{P}$ films. Upon anion formation, the molecule is planarized and orients exclusively along the [001] azimuth due to its electrostatic bond to the substrate. The presence of charged molecules hinders the mobility of the twisted neutral molecules [38]; therefore, when approximately $30 \%$ of the molecules are charged, a transition occurs and the neutral molecules also wet the surface. The conformational change induced by charge transfer leads to the final pinning work function being higher than the critical work function, as the latter is related to the electron affinity of the neutral twisted molecule, while the former is associated with the EA of the planar conformation. Increasing $\mathrm{MgO}$ thickness decreases the EA due to reduced polarization [20]. The $\Phi_{\text {crit }}$ is reduced to such a low value that systematic thickness-dependent studies of charge transfer to $6 \mathrm{P}$ could not be performed.

On dielectric interlayers, a simple measurement of the work function before and after the adsorbate overlayer growth is perhaps the most telling result with regard to electronic and physical coupling. If there is only a small reduction of $\Phi$, then the system is in the vacuum level alignment regime and there is no charge transfer. If there is any significant change in $\Phi$, then the system is in the Fermi level pinning regime with equilibrium achieved by a balance between charged and neutral adsorbates in the first monolayer. An increase in $\Phi$, as in the examples here, implies a charge transfer from the underlying substrate to the LUMO while a decrease in $\Phi$ would indicate integer charge transfer from the HOMO to the substrate. The mode of charge transfer is primarily determined by the electron affinity and ionization potential levels and their relation to the Fermi level of the substrates. This is set by the dielectric/substrate work function.

It is important to emphasize that once in the Fermi level pinning regime, both charged and neutral species will co-exist on the dielectric. This has not been recognized until now because many of the standard techniques cannot easily distinguish between charged and neutral species. For reasonably large molecules with delocalized frontier orbitals, the integer charge is spread over many atoms and the resulting chemical shift will be too small to be seen with X-ray photoelectron spectroscopy [20]. Similarly, the LEED technique will probably not be sensitive to the charge state per se, although differences in the molecular orientation and in the long-range order might occur. With a thorough analysis and molecular orbital resolution, it should be possible to distinguish them with scanning probe techniques. Generally, however, studies with orbital resolution tend to focus on very low coverages, where only charged species will be present.

\section{Experimental}

All experiments were performed under ultrahigh vacuum (UHV) conditions with a base pressure of $\leq 3 \times 10^{-10} \mathrm{mbar}$. The $\mathrm{Ag}(100)$ crystal was cleaned by cycles of $\mathrm{Ar}^{+}$sputtering and annealing at $500{ }^{\circ} \mathrm{C}$. $\mathrm{MgO}(100)$ films were grown by $\mathrm{Mg}$ evaporation in an oxygen environment. The $\mathrm{Mg}$ fluxes used were on the order of $1 \AA /$ min as monitored by a quartz microbalance. The $\mathrm{MgO}$ deposition was done at a temperature of $270{ }^{\circ} \mathrm{C}$ and at an $\mathrm{O}_{2}$ pressure of $10^{-6}$ mbar, followed by slow cooling (approximately $2.5^{\circ} \mathrm{C} / \mathrm{min}$ ). This is the accepted procedure that provides epitaxial $\mathrm{MgO}(100)$ films with high structural quality [19,34]. One $\mathrm{ML}$ of $\mathrm{MgO}$ is defined as a single atomic layer (i.e., half a unit cell of crystalline bulk $\mathrm{MgO}$ ), corresponding to a thickness of $2.105 \AA$. Work functions, measured from the secondary electron cutoff in the photoemission, could be reduced by annealing in UHV or further Mg exposure while annealing. The work function could be increased by $\mathrm{O}_{2}$ exposure $(5 \times$ $10^{-7}-2 \times 10^{-4}$ mbar) at moderate temperatures. Monitoring the work function during this post-preparation allowed for a close control of this parameter. para-Sexiphenyl (6P) was deposited at a rate of $0.8-3 \AA / m i n$ at RT. When the $6 \mathrm{P}$ exposure is given in Angstroms, this refers to the microbalance reading with a density of $1 \mathrm{~g} / \mathrm{cm}^{3}$. After conducting the ARUPS measure- 
ments on $6 \mathrm{P} / \mathrm{MgO}(100) / \mathrm{Ag}(100), 6 \mathrm{P}$ was desorbed from the substrate by flashing to $250{ }^{\circ} \mathrm{C}$. Then, the work function of the substrate was modified as desired for the next $6 \mathrm{P}$ preparation. This allowed the experiments to be performed on a $\mathrm{MgO}$ film with the exact same thickness but with different work functions.

The photoemission tomography experiments were conducted at the Metrology Light Source insertion device beamline of the Physikalisch-Technische Bundesanstalt using the toroidal electron spectrometer. The ARUPS experiments were performed with a small hemispherical sector electron analyzer mounted on a goniometer (VG-ADES400) with a He I discharge lamp as photon source. Details of the PT and ARUPS setups as well as the identification of molecular orbitals can be found in work by Hollerer et al. [14]. The intensity values of the molecular photoemission features were determined by subtracting the background, measured prior to the adsorption, from the molecules and determining the area of the peak measured in the direction of the maximum emission intensity. At the $\mathrm{He} \mathrm{I}$ of $21.22 \mathrm{eV}$, these were at $50^{\circ}$ and $45^{\circ}$ with respect to the surface normal along the [001] azimuthal direction for the LUMO and the HOMO, respectively.

The wetting parameter quantifies the spectral changes reflecting the wetting of $6 \mathrm{P}$. It is calculated from the intensity of the $\mathrm{MgO}$ valence band peak prior to/after $6 \mathrm{P}$ deposition $\left(I_{\mathrm{MgO}} / I_{\mathrm{MgO}}\right)$ and the corresponding intensities $\left(I_{6 \mathrm{P}} / I_{6 \mathrm{P}}\right)$ in the energy range (between $14 \mathrm{eV}$ and $6 \mathrm{eV}$ below $E_{\mathrm{F}}$, normal emission) where the emission of the $6 \mathrm{P}$ sigma-orbitals is found, as

$$
\frac{I_{\mathrm{MgO}}}{I_{\mathrm{MgO}}{ }^{\prime}} \frac{I_{6 \mathrm{P}^{\prime}}}{I_{6 \mathrm{P}}}-1
$$

To assess the desorption dynamics, the samples were heated manually by a filament behind the crystal at a very low heating rate of approximately $0.1{ }^{\circ} \mathrm{C} / \mathrm{s}$ (on average).

STM measurements were performed at $-193{ }^{\circ} \mathrm{C}$ with a Createc low-temperature STM using electrochemically etched tungsten tips. The bias voltage was applied to the sample.

\section{Acknowledgements}

We would like to thank Hendrik Kaser (PTB, Germany) and John Riley (La Trobe University, Australia) for their technical support.

\section{Funding}

This work was funded by the Austrian Science Fund (FWF) (projects P27649-N20, P27427-N20 and I 3731) and the Deutsche Forschungsgemeinschaft (DFG) (projects Po 2226/2-1 and Ri 804/8-1).

\section{ORCID ${ }^{\circledR}$ iDs}

Georg Koller - https://orcid.org/0000-0001-7741-2394 Xiaosheng Yang - https://orcid.org/0000-0002-7632-0401 Anja Haags - https://orcid.org/0000-0001-9117-8805 Serguei Soubatch - https://orcid.org/0000-0002-1455-0260 Alexander Gottwald - https://orcid.org/0000-0003-2810-7419 Peter Puschnig - https://orcid.org/0000-0002-8057-7795 Martin Sterrer - https://orcid.org/0000-0001-9089-9061 Michael G. Ramsey - https://orcid.org/0000-0003-0523-1994

\section{References}

1. Repp, J.; Meyer, G.; Stojković, S. M.; Gourdon, A.; Joachim, C. Phys. Rev. Lett. 2005, 94, 026803. doi:10.1103/physrevlett.94.026803

2. Wu, S. W.; Ogawa, N.; Nazin, G. V.; Ho, W. J. Phys. Chem. C 2008, 112, 5241-5244. doi:10.1021/jp7114548

3. Swart, I.; Sonnleitner, T.; Repp, J. Nano Lett. 2011, 11, 1580-1584. doi:10.1021/nl104452x

4. Pacchioni, G.; Giordano, L.; Baistrocchi, M. Phys. Rev. Lett. 2005, 94, 226104. doi:10.1103/physrevlett.94.226104

5. Giordano, L.; Baistrocchi, M.; Pacchioni, G. Phys. Rev. B 2005, 72, 115403. doi:10.1103/physrevb.72.115403

6. Giordano, L.; Pacchioni, G. Phys. Chem. Chem. Phys. 2006, 8 , 3335-3341. doi:10.1039/b604288k

7. Sterrer, M.; Risse, T.; Heyde, M.; Rust, H.-P.; Freund, H.-J. Phys. Rev. Lett. 2007, 98, 206103. doi:10.1103/physrevlett.98.206103

8. Sterrer, M.; Risse, T.; Martinez Pozzoni, U.; Giordano, L.; Heyde, M.; Rust, H.-P.; Pacchioni, G.; Freund, H.-J. Phys. Rev. Lett. 2007, 98, 096107. doi:10.1103/physrevlett.98.096107

9. Gonchar, A.; Risse, T.; Freund, H. J.; Giordano, L.; Di Valentin, C.; Pacchioni, G. Angew. Chem., Int. Ed. 2011, 50, 2635-2638. doi:10.1002/anie.201005729

10. Starr, D. E.; Weis, C.; Yamamoto, S.; Nilsson, A.; Bluhm, H. J. Phys. Chem. C 2009, 113, 7355-7363. doi:10.1021/jp900410v

11. Schulz, F.; Drost, R.; Hämäläinen, S. K.; Liljeroth, P. ACS Nano 2013, 7, 11121-11128. doi:10.1021/nn404840h

12. Wang, H.; Amsalem, P.; Heimel, G.; Salzmann, I.; Koch, N.; Oehzelt, M. Adv. Mater. (Weinheim, Ger.) 2014, 26, 925-930. doi:10.1002/adma.201303467

13. Yang, X.; Krieger, I.; Lüftner, D.; Weiß, S.; Heepenstrick, T.; Hollerer, M.; Hurdax, P.; Koller, G.; Sokolowski, M.; Puschnig, P.; Ramsey, M. G.; Tautz, F. S.; Soubatch, S. Chem. Commun. 2018, 54 , 9039-9042. doi:10.1039/c8cc03334j

14. Hollerer, M.; Lüftner, D.; Hurdax, P.; Ules, T.; Soubatch, S.; Tautz, F. S.; Koller, G.; Puschnig, P.; Sterrer, M.; Ramsey, M. G. ACS Nano 2017, 11, 6252-6260. doi:10.1021/acsnano.7b02449

15. Hofmann, O. T.; Rinke, P.; Scheffler, M.; Heimel, G. ACS Nano 2015, 9, 5391-5404. doi:10.1021/acsnano.5b01164

16. Cho, S. B.; Yun, K.-H.; Yoo, D. S.; Ahn, K.; Chung, Y.-C. Thin Solid Films 2013, 544, 541-544. doi:10.1016/j.tsf.2012.12.072

17. Jaouen, T.; Aebi, P.; Tricot, S.; Delhaye, G.; Lépine, B.; Sebilleau, D.; Jézéquel, G.; Schieffer, P. Phys. Rev. B 2014, 90, 125433. doi:10.1103/physrevb.90.125433

18. Jaouen, T.; Tricot, S.; Delhaye, G.; Lépine, B.; Sébilleau, D.; Jézéquel, G.; Schieffer, P. Phys. Rev. Lett. 2013, 111, 027601. doi:10.1103/physrevlett.111.027601

19. Pal, J.; Smerieri, M.; Celasco, E.; Savio, L.; Vattuone, L.; Ferrando, R.; Tosoni, S.; Giordano, L.; Pacchioni, G.; Rocca, M. J. Phys. Chem. C 2014, 118, 26091-26102. doi:10.1021/jp507718n 
20. Hurdax, P.; Hollerer, M.; Puschnig, P.; Lüftner, D.; Egger, L.; Ramsey, M. G.; Sterrer, M. Adv. Mater. Interfaces 2020, 7, 2000592. doi:10.1002/admi.202000592

21. Hollerer, M.; Pachmajer, S.; Lüftner, D.; Butej, B.; Reinisch, E.-M.; Puschnig, P.; Koller, G.; Ramsey, M. G.; Sterrer, M. Surf. Sci. 2018, 678, 149-156. doi:10.1016/j.susc.2018.04.017

22. Koller, G.; Berkebile, S.; Krenn, J. R.; Tzvetkov, G.; Hlawacek, G.; Lengyel, O.; Netzer, F. P.; Teichert, C.; Resel, R.; Ramsey, M. G. Adv. Mater. (Weinheim, Ger.) 2004, 16, 2159-2162. doi:10.1002/adma.200400276

23. Koller, G.; Berkebile, S.; Oehzelt, M.; Puschnig, P.; Ambrosch-Draxl, C.; Netzer, F. P.; Ramsey, M. G. Science 2007, 317, 351-355. doi:10.1126/science.1143239

24. Oehzelt, M.; Grill, L.; Berkebile, S.; Koller, G.; Netzer, F. P.; Ramsey, M. G. ChemPhysChem 2007, 8, 1707-1712. doi:10.1002/cphc.200700357

25. Wagner, M.; Berkebile, S.; Netzer, F. P.; Ramsey, M. G. ACS Nano 2015, 9, 12070-12078. doi:10.1021/acsnano.5b05013

26. Baker, K. N.; Fratini, A. V.; Resch, T.; Knachel, H. C.; Adams, W. W.; Socci, E. P.; Farmer, B. L. Polymer 1993, 34, 1571-1587. doi:10.1016/0032-3861(93)90313-y

27. Puschnig, P.; Ramsey, M. G. Photoemission Tomography: Valence Band Photoemission as a Quantitative Method for Investigating Molecular Films. In Encyclopedia of Interfacial Chemistry; Wandelt, K., Ed.; Elsevier: Amsterdam, Netherlands, 2018; pp 380-391. doi:10.1016/b978-0-12-409547-2.13782-5

28. Puschnig, P.; Berkebile, S.; Fleming, A. J.; Koller, G.; Emtsev, K.; Seyller, T.; Riley, J. D.; Ambrosch-Draxl, C.; Netzer, F. P.; Ramsey, M. G. Science 2009, 326, 702-706. doi:10.1126/science.1176105

29. Berkebile, S.; Koller, G.; Fleming, A. J.; Puschnig, P.; Ambrosch-Draxl, C.; Emtsev, K.; Seyller, T.; Riley, J.; Ramsey, M. G. J. Electron Spectrosc. Relat. Phenom. 2009, 174, 22-27. doi:10.1016/j.elspec.2009.04.001

30. Grimm, M.; Metzger, C.; Graus, M.; Jugovac, M.; Zamborlini, G.; Feyer, V.; Schöll, A.; Reinert, F. Phys. Rev. B 2018, 98, 195412. doi:10.1103/physrevb.98.195412

31. Reinisch, E. M.; Puschnig, P.; Ules, T.; Ramsey, M. G.; Koller, G. Phys. Rev. B 2016, 93, 155438. doi:10.1103/physrevb.93.155438

32. Reinisch, E. M.; Ules, T.; Puschnig, P.; Berkebile, S.; Ostler, M.; Seyller, T.; Ramsey, M. G.; Koller, G. New J. Phys. 2014, 16, 023011. doi:10.1088/1367-2630/16/2/023011

33. Brédas, J. L.; Thémans, B.; Fripiat, J. G.; André, J. M.; Chance, R. R. Phys. Rev. B 1984, 29, 6761-6773. doi:10.1103/physrevb.29.6761

34. Pal, J.; Smerieri, M.; Celasco, E.; Savio, L.; Vattuone, L.; Rocca, M. Phys. Rev. Lett. 2014, 112, 126102. doi:10.1103/physrevlett.112.126102

35. Subramanian, M. A.; Shannon, R. D.; Chai, B. H. T.; Abraham, M. M.; Wintersgill, M. C. Phys. Chem. Miner. 1989, 16, 741-746. doi:10.1007/bf00209695

36. Prada, S.; Martinez, U.; Pacchioni, G. Phys. Rev. B 2008, 78, 235423. doi:10.1103/physrevb.78.235423

37. Heimel, G.; Salzmann, I.; Duhm, S.; Rabe, J. P.; Koch, N. Adv. Funct. Mater. 2009, 19, 3874-3879. doi:10.1002/adfm.200901025

38. Hla, S.-W.; Braun, K.-F.; Wassermann, B.; Rieder, K.-H. Phys. Rev. Lett. 2004, 93, 208302. doi:10.1103/physrevlett.93.208302

\section{License and Terms}

This is an Open Access article under the terms of the Creative Commons Attribution License (https://creativecommons.org/licenses/by/4.0). Please note that the reuse, redistribution and reproduction in particular requires that the authors and source are credited.

The license is subject to the Beilstein Journal of Nanotechnology terms and conditions: (https://www.beilstein-journals.org/bjnano)

The definitive version of this article is the electronic one which can be found at: https://doi.org/10.3762/bjnano.11.132 